\title{
Thống kê "tóe lung tung" vì chuẩn trích dẫn... phong phú quá
}

AISDL

Ngày 26 tháng 11 năm 2020

Phòng lab AISDL làm một số công việc liên quan đến thống kê scientometrics và bibliometrics.

Một thực tế có thể thấy là hiện nay có nhiều cơ sở dữ liệu cho mục tiêu nói trên, Scopus, Web of Science, PubMed, SCIELO, Google Scholar, Microsoft Academic, v.v..

Tuy nhiên, nếu nói về phổ biến và thông dụng nhất thì chắc ai cũng đồng ý là CSDL Google Scholar, https://scholar.google.com.

Ngoài việc tìm tài liệu, Google Scholar còn rất hữu ích cho việc nhận biết tài liệu nào được sử dụng nhiều, có ảnh hưởng, thông qua số lượt trích dẫn (citations), nằm ngay bên dưới bài.

Trong việc sử dụng Google Scholar có một vấn đề là khi tìm một tài liệu, đôi khi nó bị phân mảnh dữ liệu trích dẫn. Cụ thể là, vẫn một tài liệu đó, nhưng chỗ này 16 citations, nhưng rồi lại có 1 dòng khác có 3 citations, rồi có thể một dòng nữa 2 citations.

Chúng tôi gọi dân dã vấn đề này là "thống kê tóe lung tung".

Nguyên nhân thì chẳng có gì bí ẩn: Cùng một tài liệu đó, mỗi người dùng một kiểu trích dẫn. Đó là chưa kể, khi trích dẫn thì vì lý do kỹ thuật, thiếu một hai ký tự, rồi thừa một hai ký tự, hay chuyển vị trí một vài thông tin, v.v.. Thế là hệ thống nhận biết vô cùng thông minh của Google cho rằng đó là hai tài liệu khác nhau, và họ bắt đầu đếm citations cho từng đối tượng một cách riêng rẽ.

Vấn đề này càng dễ mắc phải hơn với các bản Working Paper (WP). Có thể đoán biết lý do là vì WP tuy có đánh số, và có ngày tháng, nhưng quy định về việc trích dẫn khó tuân thủ.

Ví dụ, một hệ thống có thể quy định cách trích dẫn cụ thể nào đó. Nhưng lại có tới cả chục chuẩn trích dẫn khác nhau. Hướng dẫn đó rõ ràng cho loại này, nhưng với các hệ khác thì vẫn thành không có chuẩn.

Chính việc thiếu đi chuẩn dẫn chiếu tài liệu, dẫn tới có người thì thêm thông tin cho kỹ, lại có người bỏ bớt thông tin cho gọn. (Đó là còn bỏ qua việc có thể gõ sai thông tin.)

Và thế là, tác giả "thu nhận" được dăm ba phiên bản khác nhau của cùng một văn bản trên Google Scholar. Cũng chính vì lẽ đó, thống kê trích dẫn cũng "tóe lung tung" cả.

Dưới đây là một ví dụ lấy từ một tác giả thuộc chính AISDL, trên một văn bản được sử dụng tương đối thường xuyên trong 6 năm vừa qua. 
Trong tựa đề văn bản có cụm từ "Vietnam's political economy". Sử dụng cụm từ này có ngoặc kép để tìm chính xác văn bản qua CSDL Google Scholar thì trả về kết quả như trong Hình 1.

Hình 1. Tìm kiếm "Vietnam's political economy" trong Google Scholar

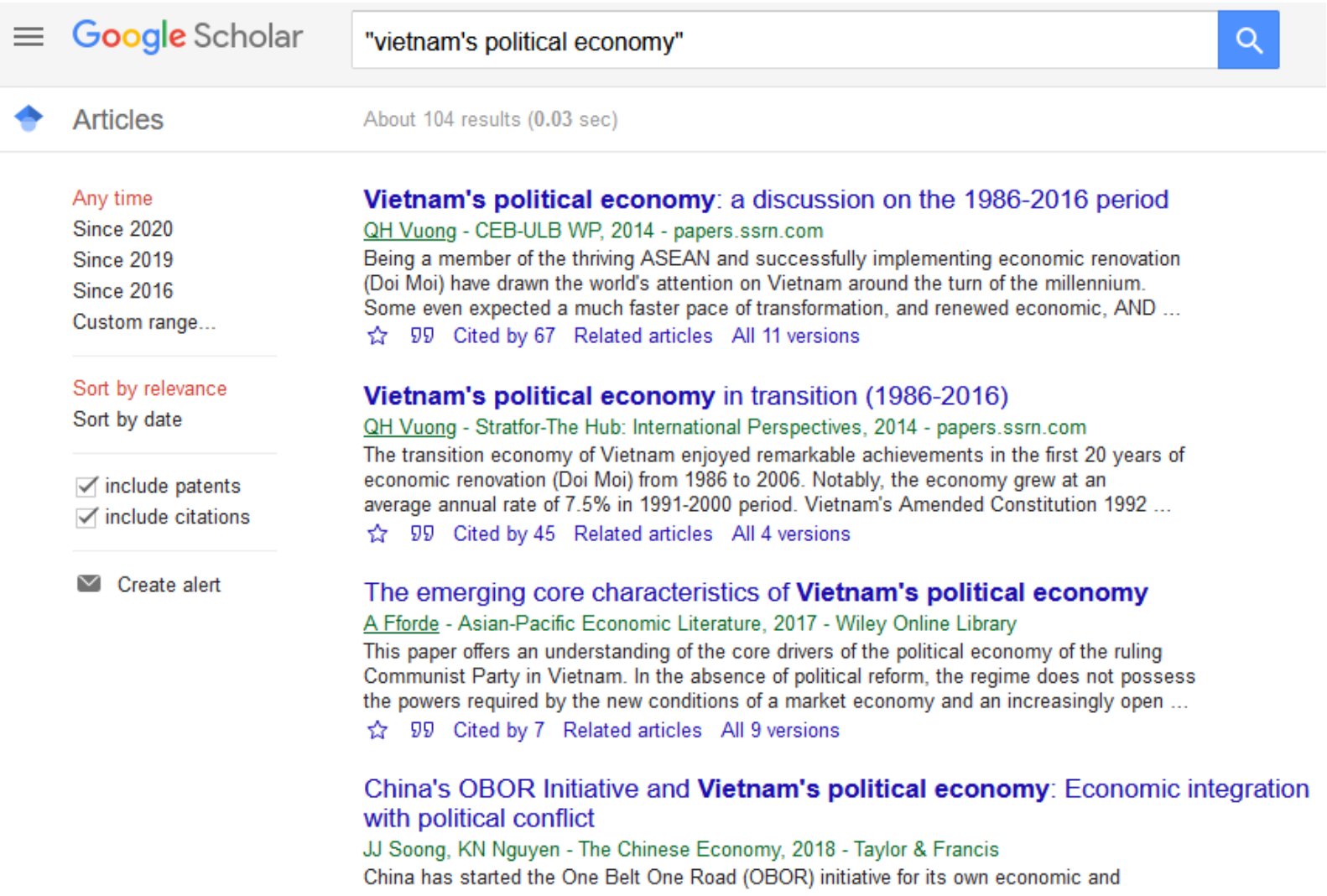

https://scholar.google.com/scholar?\&q=\%22vietnam $\% 27 s+$ political+economy\%22\&btnG=

Xuất hiện ở trang trả kết quả tìm kiếm đầu tiên là hai văn bản của cùng tác giả, trong đó một bản dài hơn 30 trang (chính là bản WP gốc [1]).

Kết quả kia trỏ tới bản tóm lược đăng trên trang chuyên xuất bản các bài phân tích địa chính trị quốc tế Stratfor Worldview [2].

Tuy nhiên, vấn đề được chỉ ra dễ thấy trong Hình 2, Hình 3.

Trong Hình 2, chẳng cần đợi đâu xa xôi, mà chỉ kéo xuống cuối trang 1 của kết quả tìm kiếm, cái sự "tóe lung tung" đã bắt đầu phát tác.

Bài [2] được tách thành hai văn bản khác nhau! Trong đó có một entry là do người viết nào đó đánh sai năm xuất bản bài, 2017. Đồng thời họ cũng ghi trật tự tên tác giả sai khác đi so với bài báo! 
Còn entry kia thì không rõ vì lý do gì mà tên nơi xuất bản đã nhảy lên tựa đề bài. Có lẽ vì thế mà bài đã được hiểu thành một bài khác (có tựa đề khác).

Hình 2. Tóe lung tung 1
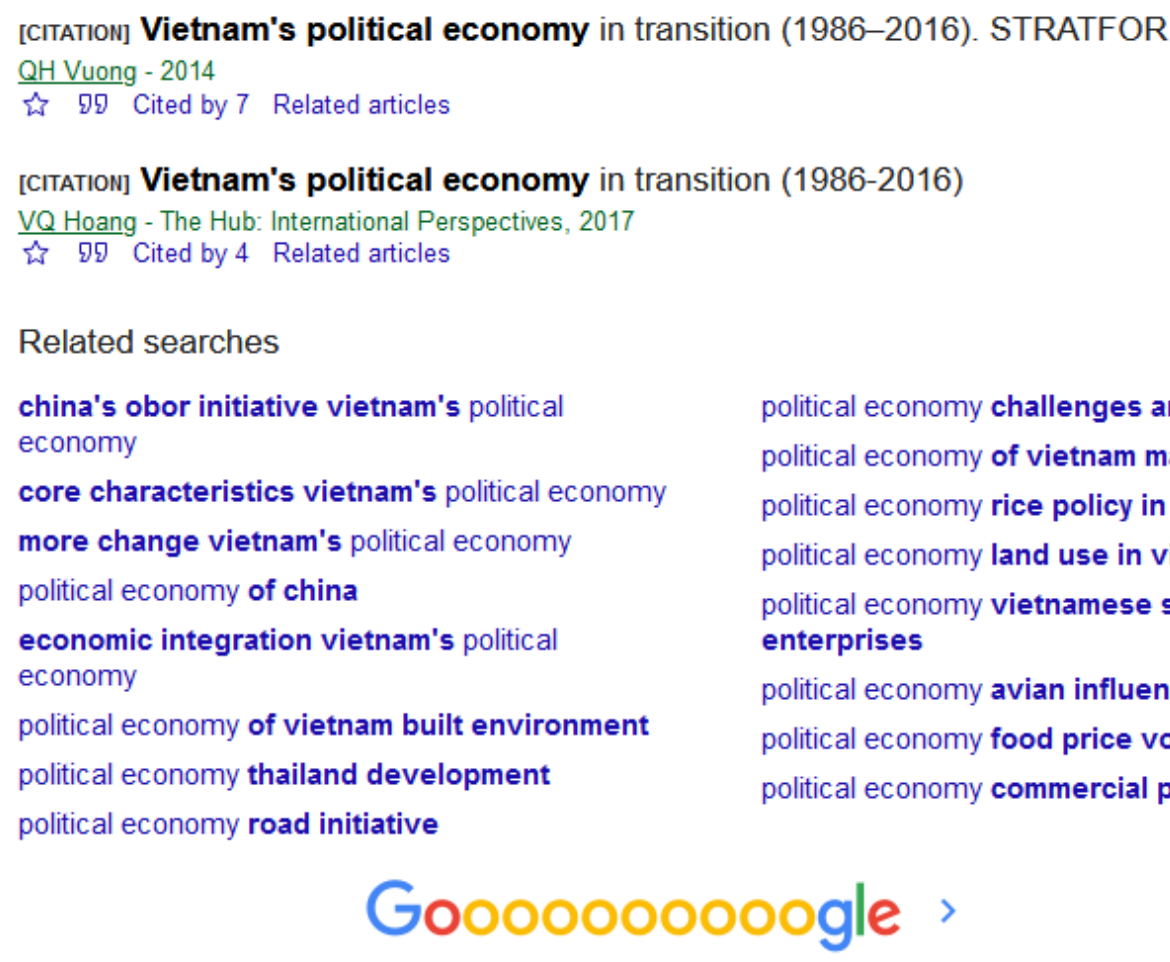

$\begin{array}{llllllllll}1 & 2 & 3 & 4 & 5 & 6 & 7 & 8 & 9 & 10\end{array}$

Next

Tóe lung tung ngay tại cuối trang tìm kiếm đầu tiên

Nhưng đó chưa phải là tất cả. Sang tới trang kết quả tìm kiếm thứ hai, xem Hình 3, thì thấy có tới 3 dòng khác nhau của văn bản thứ nhất [1].

Khi nhìn vào thông tin "metadata" của từng phiên bản mà Google ghi lại này thì thấy chúng rất khác nhau, mỗi dòng ghi một kiểu.

Tuy vậy, có thể quan sát thấy, nguyên nhân chính của việc tách rời này là tựa đề văn bản được ghi nhận hoàn toàn khác nhau. Đây đơn giản là cách ghi nhận văn bản khi trích dẫn của người viết, dựa trên thông tin nhận có được từ văn bản gốc, nhưng đặt xuống thành dòng chữ khác nhau.

Điều kỳ diệu là 3 văn bản “khác nhau” này có cùng một lượng trích dẫn là 2.

Hình 3. Tóe lung tung 2 
Google Scholar

\section{Articles}

Any time

Since 2020

Since 2019

Since 2016

Custom range.

Sort by relevance

Sort by date

include patents

$\checkmark$ include citations

Create alert "vietnam's political economy"
Page 2 of about 104 results $(0.04 \mathrm{sec})$

[PDF] Political economy of Vietnam: Market reform, growth, and the state JM Steinfeld, KV Thai - Maryland Series in Contemporary ..., 2013 - digitalcommons.odu.edu 68 R I. INTRODUCTION The evolution of Vietnam's political economy is unlike that of any other nation in the world today ... Page 12. Ijciprod01/productn\ALAOP\13-3LAOP301.txt unknown Seq: 11 8-OCT-13 13:44 POLITICAL ECONOMY OF VIETNAM 11

约 59 Cited by 5 Related articles All 6 versions

[CITATION] Vietnam's Political Economy: A Discussion on the 1986-2016 Period (May 21, 2014)

QH Vuong - CEB-ULB Working Paper, 2014

ऽ 59 Cited by 2

[CITATION] Vietnam's political economy: a discussion on the 1986-2016 period (No. 14-010)

QH Vuong - ULB--Universite Libre de Bruxelles, 2014

is 50 Cited by 2 Related articles

[CITATION] Vietnam's political economy: A discussion on the 1986-2016 period (Report No. CEB-ULB WP N0 14/010)

QH Vuong - Brussels: Université Libre de Bruxelle, 2014

is 59 Cited by 2 Related articles

Competing legacies: rupture and continuity in Vietnamese political economy

G Sasges, S Cheshier - South East Asia Research, 2012 - Taylor \& Francis

At what point is it appropriate to throw out assumptions of rupture and the highly imperfect

Trang kết quả thứ 2 tiếp tục tóe loe ra nữa...

Như vậy, từ hai văn bản gốc [1,2] nay đã được cộng thêm 5 Google Scholar entries nữa, 3 cho tài liệu [1] và 2 cho tài liệu [2].

Quá trình này vẫn chưa kết thúc ở trang kết quả thứ 2 .

Trang kết quả thứ 3 được cho trong Hình 3. Ta lại thấy xuất hiện thêm 2 entries mới của cùng bản WP [1], với thông tin rất khác nhau trên tiêu đề.

Rõ ràng, kết quả trả ra này cho thấy cách viết thông tin bài trích dẫn của các tác giả khi sử dụng tài liệu hết sức khác biệt, một phần do chuẩn, nhưng phần khác còn do chính các thông tin mà người viết lựa chọn đưa vào sử dụng.

(Điều thú vị là, trong Hình 4, ngay bên dưới cũng có một bài xuất hiện do nó chứa thông tin trích dẫn tài liệu và qua đó có thể thấy một cách ghi thông tin.)

Như vậy, bây giờ tỷ số trội lên giữa [1]:[2] là 5:2. Phần tóe lung tung vượt trội đang ngả về phía WP [1]. Điều này cũng phản ánh bản dài đầy đủ được sử dụng nhiều hơn. Trên thực tế, nhìn hai phiên bản gốc (có URL tải tài liệu và có lượng trích dẫn lớn nhất), thì tỷ số giữa [1]:[2] đang là 67:45. 


\section{Hình 4. Tóe lung tung 3}

[CITATION] Vietnam's political economy: a discussion on the 1986-2016 period, ULB-- Universite Libre de Bruxelles, paper

QH Vuong - 2014

is 59 Cited by 2 Related articles

[CITATION] Vietnam's political economy: a discussion on the 1986-2016 period. CEBULB WP N 14/010; Université Libre de Bruxelles

$\mathrm{QH}$ Vuong - 2014

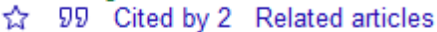

Current Situation of Corruption Offenses and Measures for Improvement of Anti-

Corruption Effectiveness in Vietnam's Economy

TT Thao - 2nd International Scientific and Practical Conference ..., 2020 - atlantis-press.com

VI 3(4) (2009) 54-78. The Icfai University Press, September-December 2009. DOI:

10.2139/ssrn.1442384. [9] QH Vuong, Vietnam's Political Economy: A Discussion on the

1986-2016 Period (May 21, 2014). CEB-ULB WP N 14/010 (2014). DOI: 10.2139/ssrn.2439809 ...

^ 90 All 3 versions $\gg$

The Vietnamese economy: a remarkable development record

NR Harispe - 2018 - philpapers.org

... inflation (22). References: (1) Vuong, Quan Hoang, 2014. "Vietnam's Political Economy

in Transition (1986-2016)", Stratfor Worldview, https://worldview.stratfor.com/article/

vietnams-po... (page consulted on March 9, 2018) Page 3. (2 ..

々 99 All 3 versions

[CITATION] Cnnmllan Cataloguing in Publication Data

PM Evans

ک 59 Related articles

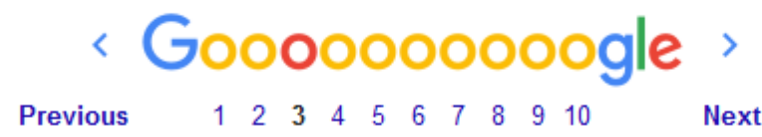

Trang kết quả 3 vẫn "tóe lung tung"

Sơ qua như vậy cũng có thể thống kê nhanh số lượt trích dẫn khả dĩ của [1] là 77 (67+5×2), và của [2] là 66 (tức 45+7+4).

Mở rộng phạm vi, chúng tôi nhận thấy "nạn nhân" còn bao gồm cả các ấn phẩm xuất bản tạp chí có đầy đủ lệ bộ về số volume, số issue, số trang, DOI và được chỉ mục hóa chuẩn mực. Dù thế, vẫn gặp nhiều trường hợp một văn bản được ghi thành 2, 3 thậm chí 5 hay hơn nữa, với thống kê trích dẫn phân mảnh.

\section{References}

[1] Vuong, Q. H. (2014). Vietnam's Political Economy: A Discussion on the 1986-2016 Period. CEB Working Papers Series, №14-010, Université Libre de Bruxelles, Brussels, Belgium.

[2] Vuong, Q. H. (2014). Vietnam's Political Economy in Transition (1986-2016). Stratfor - The Hub: International Perspectives (May 27, 2014). 